\title{
Entwicklung von Polyurethan-Beschichtungssystemen auf der Basis von Recyclat-Polyolen aus Polyurethan-Weichschaumstoffen
}

\author{
Gerhard Behrendt, Hannelore Huth, Svetlana Ivanyi, Hagen Koch, Martin Pohl, \\ Karl-Heinz Schmidt, Oskar Stürmer
}

\section{Einleitung}

Gegenwärtig wird die Hauptmenge von PolyurethanWeichschaumstoffabfällen aus Produktionsprozessen oder der Kfz-Demontage verbrannt oder deponiert. Bei diesem Typ von Kondensationspolymeren bestehen im Gegensatz zu den thermoplastischen Kunststoffen Verwertungsmöglichkeiten nur durch das direkte stoffliche Recycling, z. B. durch Flockung von Altschaumstoffen und deren direkte Verwendung oder Verklebung der Flocken, durch die energetische Verwertung (Verbrennung mit oder ohne Brikettierung) oder durch den chemischen Abbau der Makromoleküle, z. B. durch Pyrolyse, Hydrolyse, Solvolyse oder Hydrierung. Die Verfahren zur chemischen Spaltung haben gegenüber den anderen den Vorteil, dass durch sie die Makromolekülle gezielt in Oligomere oder Monomere umgesetzt werden, die dann weiter verwertet werden können.

Die Zielstellung besteht in der Synthese neuer Ausgangsstoffe für die Herstellung von Kunststoffen. In ökologischer Hinsicht ist das Ziel, bisher nicht genutzte Abfallstoffe ohne Wertminderung durch die Realisierung einer neuen Technologie wieder in den Wirtschaftskreislauf zurückzuführen; die Umweltbelastung wird durch weniger Deponierung und weniger Verbrennung geringer; durch die Bereitstellung von Recycling-Polyolen aus Weichschaumstoffen kann die Masse an Primärprodukt reduziert werden, was wiederum zur Senkung des Gesamtenergieverbrauches und zur Schonung der Ressour- cen beiträgt. Ökonomisch sollen durch die Herstellung von Spezialprodukten aus den Recyclaten bei günstigen Herstellungskosten neue Anwendungsgebiete erschlossen werden.

Die Arbeiten waren auf ein verändertes Glykolyseverfahren gerichtet, durch das aus den PUR-Weichschaumstoffen neue Polyole hergestellt werden, die im Sinne des o. g. Ziels zu elastischen Produkten, insbesondere Beschichtungen und Vergussmassen, formuliert werden und über die Formulierung mit Polyisocyanaten zu hochwertigen Polyurethanen verarbeitet werden können. Damit werden PUR-Weichschaumstoffe im Sinne der Kreislaufwirtschaft recycelt, jedoch nicht zu den ursprünglichen Produkten, sondern zu modernen, neuen Materialien mit einer wesentlich höheren Wertschöpfung.

Dieses Ziel soll durch eine neue Glykolysevariante durch Umsetzung der PUR-Weichschaumstoffe mit einem Reaktionsgemisch aus höheren Glykolen (Molmasse über 150 $\mathrm{g} / \mathrm{Mol}$ ) und aliphatischen primären und/oder sekundären Aminen im Temperaturbereich $120-180^{\circ} \mathrm{C}$ erreicht werden. Die daraus hergestellten Recyclatpolyole mit unterschiedlichen Eigenschaften (Hydroxylzahl, Viskosität, Reaktivität) werden mittels speziell entwickelter Formulierungen zu den Zielpolyurethanen umgesetzt und im Labor auf dem Mathis ${ }^{\circledR}$ Labcoater zu Beschichtungen verarbeitet und durch physikalische Messungen sowie mechanische Prüfungen charakterisiert.

\section{Bisherige Arbeiten}

Die Umsetzung der Polyisocyanate mit alkoholischen Hydroxylgruppen bzw. Wasser als wesentliche Bildungsreaktionen in den Polyurethanen fuihrt zu Harnstoff- bzw. Urethangruppen nach folgenden Reaktionsschemata:

Reaktion von Isocyanaten R-NCO, z. B. 2,4 -Toluylendiisocyanat, mit Alkoholen -OH:<smiles>[R]CCCOC(=O)Nc1cccc(NC(=O)OCCP)c1C</smiles>

Reaktion von R-NCO mit Wasser<smiles></smiles> 
und der entstehenden Aminogruppe in einer sehr schnellen Reaktion weiter mit 2,6-Toluylendiisocyanat zu (Poly)harnstoffen:<smiles>CCCCNc1cccc(NC(=O)Nc2cccc(O[N+](=O)[O-])c2C)c1C</smiles>

Die Spaltung der Polyurethane ist eine Folge von Reaktionsschritten, die auf den besonderen Eigenschaften der Reaktionsprodukte in den Polyurethan-Schaumstoffen beruhen. Durch die Umsetzung der Reaktionsprodukte mit Solvolysereagenzien entstehen je nach der Art des Reagenzes unterschiedliche Reaktionsprodukte. Bei der einfachen Glykolyse werden die Urethangruppen (Carbamidsäureester - $\mathrm{NH}-\mathrm{CO}-\mathrm{O}-$ ) einfach umgeestert nach:

Urethan-Spaltung mit - OH<smiles>[R8]CCCCc1cccc(NC(=O)OCCC[R7])c1C</smiles>

Dabei entstehen als Reaktionsprodukte Urethane mit dem als Solvolysereagenz eingesetzten Glykol als Veresterungskomponente und die als Polyolkomponente im Polyurethan verwendeten Polyester- bzw. Polyetheralkohole. Als Nebenreaktion können durch die Glykole in Abhängigkeit von ihrer Nucleopbilie auch Harnstoffgruppen (-NH-CO-NH-) gespalten werden:

Harnstoff-Spaltung mit -OH

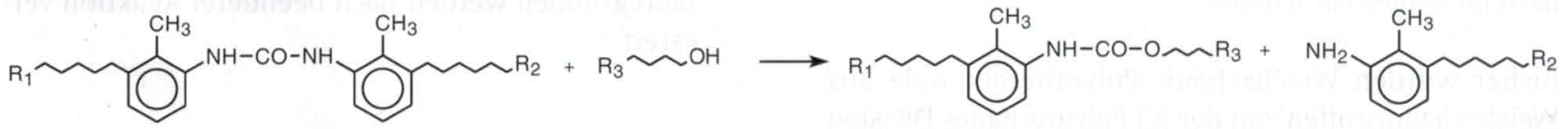

Bei dieser Umsetzung entstehen zwangsläufig primäre aromatische Amine, die wegen ihrer toxikologischen Eigenschaften unerwünscht sind. Durch die Verwendung von aliphatischen Aminen zusammen mit den Glykolen reagieren diese vorzugsweise mit den Urethangruppen unter Bildung von araliphatischen Harnstoffen:

Urethan-Spaltung mit aliphatischen Aminen $\mathrm{R}_{-} \mathrm{NH}_{2}$<smiles>CC=CC(=CC)CCCCBr</smiles>

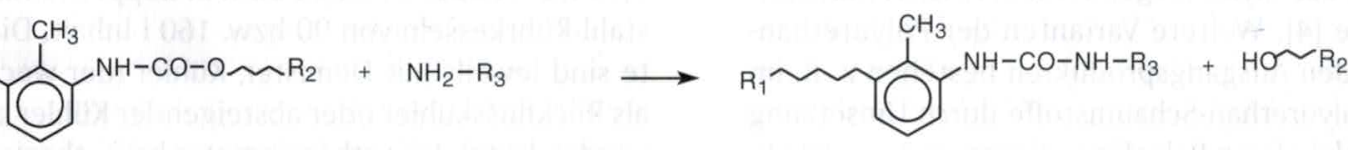

Bei diesem Reaktionsweg entstehen formal keine primären aromatischen Amine. Die Spaltung der Harnstoffe kann ebenfalls durch Carbonsäuregruppen oder deren Anhydride erfolgen:

Reaktionen an der Harnstoffgruppe mit Carbonsäuregruppen R-COOH:

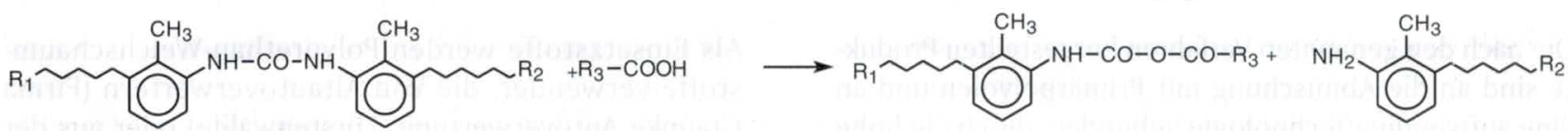<smiles>[R9]OC(=O)Nc1cccc(CCCCBr)c1C</smiles>

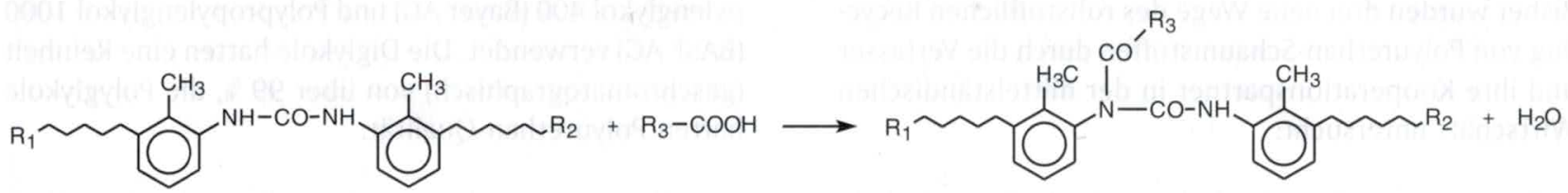

Bei dieser Umsetzung entstehen Acylharnstoffe, die im weiteren Reaktionsverlauf abgebaut werden können und deshalb ebenfalls nicht erwünscht sind. Carbonsäuregruppen sind jedoch in den hier eingesetzten Produkten nur in geringem Maße enthalten. 
Die Polyole aus der Glykolyse von Mischabfällen aus Autositzen, d. h. Polyurethan-Weichschaumstoff, Polyamid- und Polyesterfasern [1], lassen sich sowohl im Handversuch als auch mit der Maschine ohne Zusatz von Primärpolyol verschäumen. Die Untersuchungen zur Formulierung von Polyurethanen ausschließlich aus Recyclat-Polyolen wurden erfolgreich abgeschlossen. Damit wurde nachgewiesen, dass die Recyclat-Polyole als alleinige Polyol-Komponenten zur Herstellung von Polyurethan-Hartschaumstoffen geeignet sind [2]. Bei der Verschäumung zu PUR-Hartschaumstoffen wurde bei bestimmten Recyclat-Polyolen beobachtet, dass bei ausgewählten Katalysatoren und unter ansonsten unüblichen Bedingungen der PUR-Hartschaumherstellung Produkte mit elastischen Eigenschaften erhalten werden können. Diese Schaumstoffe hatten jedoch zunächst eine unzulängliche Schaumstruktur, so dass eine Weiterentwicklung des Verfahrens der Polyolherstellung erforderlich schien, wenn die Zielrichtung elastische Polyurethane sein sollte. Umfangreiche Untersuchungen mit Recyclat-Polyolen zur Formulierung von Polyurethan- und Polyisocyanurat-Hartschaumstoff-Systemen wurden dazu durchgefuihrt. Dazu wurden Recyclat-Polyole verwendet, die analog denen aus der Versuchsproduktion der BASF AG nach [3] hergestellt wurden.

Bisher wurden Weichschaum-Polyetheralkohole aus Weichschaumstoffen von der ICI Polyurethanes Division Ltd. (Großbritannien) durch ein Glykolyse-Redestillationsverfahren hergestellt. Bei diesem Verfahren erfolgt die Glykolyse mit einem Glykolgemisch, das nach Beendigung der Umsetzung in zwei Phasen zerfällt. Die erste Phase enthält im Wesentlichen den dem PUR-Weichschaum zugrundeliegenden Polyetheralkohol, die zweite Phase enthält das Glykolysegemisch, Urethane, Harnstoffe und Amine [4]. Weitere Varianten der PolyurethanSpaltung zu den Ausgangsprodukten bestehen z. B. im Abbau der Polyurethan-Schaumstoffe durch Umsetzung mit niedermolekularen Polyolen in Gegenwart von Wachsen oder Ölen oder durch Lösen des Polyurethan-Weichschaumstoffes in niedermolekularen Glykolen und Abtrennen eines Teils des Glykols im Vakuum sowie in ihrer Umsetzung in einem speziell entwickelten Reaktionsextruder mit daran anschließender Urethanspaltung in Gegenwart von Glykolen.

Die nach den genannten Verfahren hergestellten Produkte sind an die Abmischung mit Primärpolyolen und an eine aufwendige Technologie gebunden, durch die hohe Kosten entstehen. Demzufolge sind die Herstellungskosten für die Produkte entsprechend hoch, so dass diese Verfahren praktisch keine Chance auf technische Realisierung haben.

Bisher wurden drei neue Wege des rohstofflichen Recycling von Polyurethan-Schaumstoffen durch die Verfasser und ihre Kooperationspartner in der mittelständischen Wirtschaft untersucht:

1. Der erste Weg besteht darin, an Stelle der Glykole in der Glykolyse niedermolekulare Abfälle aus der Polyestersynthese (sog. Oligoesterkondensate) zu verwenden, um einmal die Verfahrenskosten zu senken und zum anderen einen weiteren, bisher nicht genutzten Abfallstrom stofflich zu verwerten. Darüber hinaus wird durch den höheren Aromatenanteil in den Oligomerketten dieses Produkts eine bessere thermische Stabilität der daraus hergestellten Hartschaumstoffe erreicht.

2. Der zweite Weg besteht in einer Kombination von Glykolyse und Aminolyse der Polyurethan-Schaumstoffe. Bei diesem Verfahren entstehen di- und/oder trisubstituierte Harnstoffe, die sich im Polyolgemisch lösen, und es entstehen wieder Polyetheralkohole, die für Weichschaumstoffe geeignet sind.

3. Der dritte Weg besteht in der Acidolyse von Weichschaumstoffen. Durch die Reaktion von Dicarbonsäuren und Weichschaumstoffen bilden sich Acylharnstoffe, wobei die Polyetheralkohole in ihrer ursprünglichen Form zurückerhalten werden. Bei teuren Diisocyanaten bietet sich die Abtrennung der Acylharnstoffe und die Rüickspaltung zum Diamin an; bei den Standarddiisocyanaten sollten die Acylharnstoffe im Polyolgemisch verbleiben. Überschüssige Carbonsäuregruppen werden nach beendeter Reaktion verestert.

\section{Darstellung der angewandten Methoden}

Das Recycling der Polyurethan-Weichschaumstoffe durch eine Kombination aus Glykolyse und Aminolyse zu Weichschaumpolyetheralkoholen erfolgt durch eine einstufige Reaktion in einem Sulfierkolben von jeweils 0,75 I, 2,5 I oder 6 I Inhalt bzw. in doppelwandigen Edelstahl-Ruihrkesseln von 90 bzw. 1601 Inhalt. Die Apparate sind jeweils mit Demister, Kühler (der wechselseitig als Rückflusskühler oder absteigender Kühler geschaltet werden kann), Innenthermometer bzw. -thermoelement und Propellerrüihrer ausgestattet. Die Reaktoren werden mit Stickstoff mit ca. $15 \mathrm{l} / \mathrm{h}$ beaufschlagt. Die Heizung erfolgt extern über elektrische Heizmäntel bei den Glasreaktoren bzw. durch Thermalöl mit separatem Heizaggregat (Firma Gemui, Steuerung und Programm durch die Firma Sigmar Mothes Hochdrucktechnik).

Als Einsatzstoffe werden Polyurethan-Weichschaumstoffe verwendet, die von Altautoverwertern (Firma Graunke Autoverwertung, Fürstenwalde) oder aus der Versuchsproduktion (Elastogran Schwarzheide GmbH) zur Verfuigung gestellt wurden. Das Di-n-butylamin wurde von der Merck AG bezogen. Als Glykole wurden Diethylenglykol (BASF AG), Dipropylenglykol (BASF AG), Polyethylenglykol 200 und 400 (Bayer AG), Polypropylenglykol 400 (Bayer AG) und Polypropylenglykol 1000 (BASF AG) verwendet. Die Diglykole hatten eine Reinheit (gaschromatographisch) von über $99 \%$, die Polyglykole hatten Polyurethan-Qualität.

Der allgemeine Ansatz zur Herstellung der RecyclingPolyole wurde nach [5] folgendermaßen durchgefuihrt: In das Reaktionsgefäß wird die Glykolkomponente eingewogen und unter Rühren erwärmt. Während des Auf- 
heizvorganges wird ein Teil der berechneten Menge des Amins zugesetzt. Bei einer Temperatur von $160^{\circ} \mathrm{C}$ beginnt die Zugabe der PUR-Weichschaumstoff-Flocken. Sobald die Temperatur $180^{\circ} \mathrm{C}$ erreicht, wird die restliche Menge an Amin zugegeben und weiter erwärmt. Die Schaumstoffe werden kontinuierlich bei der festgelegten Temperatur/dem Temperaturprogramm weiter zugegeben, bis die berechnete Menge eingesetzt wurde. Nach beendeter Zugabe wird das Reaktionsgemisch noch 0,2 bis 5 Stunden bei der vorgegebenen Reaktionstemperatur gerührt. Danach wird die Heizung abgeschaltet und bis zum Erreichen einer Massetemperatur von $120^{\circ} \mathrm{C}$ geruihrt. Anschließend wird in die vorbereiteten Gebinde abgefuillt. Von den Polyolen werden die charakteristischen Daten bestimmt (Viskosität, Hydroxylzahl, Aminzahl, Amingehalt mit GC, Wassergehalt).

Die Polyurethane werden aus den Recyclat-Polyolen nach [6] hergestellt. Zur Herstellung von Hartschaumstoffen wird die folgende Standard-Formulierung verwendet:

$\begin{array}{lr}\text { Polyol } & 94,68 \text { Teile } \\ \text { Wasser } & 3,02 \text { Teile } \\ \text { Polycat PC40 } & 1,43 \text { Teile } \\ \text { Dimethylcyclohexylamin } & 0,87 \text { Teile } \\ \text { Lupranat@ M } 20 \text { A } & 132,0 \text { Teile }\end{array}$

Lupranat ${ }^{\circledR}$ M 20 A und N,N-Dimethylcyclohexylamin wurden dankenswerterweise von der BASF Schwarzheide GmbH zur Verfügung gestellt. Das Polycat PC 40 ist ein Geschenk der Nitroil GmbH, Hamburg.
Zur Herstellung der Folien werden die Komponenten A und B verwendet, deren Zusammensetzung wie folgt sind:

$\begin{array}{rlr}\text { Komponente A: } & \text { Recyclat-Polyol } & 97,66 \text { Teile } \\ & \text { Acetylaceton } & 2,20 \text { Teile } \\ & \text { Vinycene } 400 & 0,14 \text { Teile }\end{array}$

Komponente B: Lupranat ${ }^{\circledR}$ M 20 A @ 90 bis 17 Teile

Die verwendete Menge des Lupranat ${ }^{\circledR}$ M 20 A ist abhängig von der Hydroxylzahl des Recyclatpolyols sowie vom eingestellten Isocyanatindex. Durch den Isocyanatindex werden die wesentlichen mechanischen Eigenschaften, darunter als ein wesentlicher Parameter die Härte der Beschichtung, eingestellt.

Die Analytik erfolgte nach dem Standard für Hydroxylzahlen [7] und dem Werkstandard der BASF AG für Aminzahlen. Die Viskosität wurde mit einem Kugelfallviskosimeter nach Höppler bestimmt. GC-MS-Untersuchungen erfolgten mit einem Hewlett-Packard-Instrument HP 5890 Serie II mit MSD 5970. Für die mechanischen Prüfungen wurde eine Prüfmaschine FPG 7/20 der Firma Kögel, Leipzig, mit automatischer Aufzeichnung von Kraft und Weg verwendet.

Die Relaxationsspektren wurden mit einem 1-Hz-Torsionspendel (Myrenne GmbH) bzw. mit einem Rheovibron DDV-II-B (Toyo Baldwin Co.) mit Aufheizraten von $2 \mathrm{~K} / \mathrm{min}$ gemessen.

\section{Ergebnisse}

Die Spaltung der Polyurethane ist eine Folge von Reaktionsschritten, die im Falle der Umsetzung mit Alkoholen und Aminen durch die individuellen Reaktionsgeschwindigkeiten in Form konkurrierender Folgereaktionen ablaufen. Die schnellere Reaktion der Urethangruppe ist die mit dem aliphatischen Amin, bei der je nach Wahl des Amins di- oder trisubstituierte araliphatische Harnstoffe entstehen (Beispiel: lineares Polyurethan auf Basis 2,6-Toluylendiisocyanat und primäres aliphatisches Amin):<smiles>[R]O[PH2+]NC(=O)Nc1cccc(PCCP[R7]OC(=O)Nc2cccc([R])c2C)c1C</smiles>

Im dargestellten Beispiel würde ein aliphatisch und aromatisch disubstituierter Harnstoff und als zweites Reaktionsprodukt der ursprünglich im Urethan verwendete Alkohol entstehen. Bei Verwendung sekundärer aliphatischer Amine entstehen folglich N,N,N'-trisubstuierte Harnstoffe. Da in den Polyurethanen bereits Polyharnstoffstrukturen aus der Reaktion des Polyisocyanats mit dem Wasser vorhanden sind, werden an diesen die Urethangruppen in endständige disubstituierte Harnstoffgruppen umgesetzt, die eine höhere Stabilität aufweisen als die diaromatisch substituierten Harnstoffe.

Das verwendete Polyisocyanat Lupranat ${ }^{\circledR}$ M 20 A der BASF AG basiert auf Anilin-Formaldehyd-Kondensaten und hat eine mittlere Isocyanatfunktionalität von ca. 3,9, so dass durch die Harnstoffbildung in den Polyurethanen verzweigte Harnstoffe entstehen, die das Polyurethan selbst in den Hartsegmenten vernetzen. Die auf diesem Wege gebildeten hochmolekularen Polyharnstoffe sind schwer löslich in den die Weichsegmente bildenden Polyolen und auch in den zur Glykolyse verwendeten Glykolen. Ihre Löslichkeit ist dabei einmal von ihrem Verzweigungsgrad sowie ihrer Molmasse und zum anderen von der Art des Glykols und seiner Kettenlänge abhängig; Glykole auf Propylenoxidbasis lösen die Polyharnstoffe besser als die auf Ethylenoxidbasis. Mit zunehmender Kettenlänge der Glykole werden die Polyharnstoffe besser löslich. Durch die Auswahl der Glykole wird folglich wesentlich die Homogenität der RecyclatPolyole bestimmt. 
Die Glykole selbst reagieren mit den Urethangruppen im Sinne einer Umesterung, bei der die kurzkettigen Diole (die im Überschuss verwendet werden) die langkettigen Polyetheralkohole ersetzen. Diese Reaktion ist wie jede Umesterung eine Gleichgewichtsreaktion. Als Nebenreaktion findet eine Spaltung von Harnstoffgruppen durch die Hydroxylgruppen statt, bei der die Hydroxylgruppen als nucleophile Reagenzien wirken und durch diese Spaltung primäre aromatische Amine bilden. Eine kurze Reaktionszeit mit wenig nucleophilen Glykolen ist für die Herstellung hochwertiger Recyclat-Polyole erwünscht. Als optimal wurde von uns Dipropylenglykol bzw. eine Mischung aus Dipropylen- und Hexapropylenglykol gefunden. Die folgende Tabelle gibt die Einsatzmengen und einige Eigenschaften von RecyclatPolyolen an:

\begin{tabular}{lllll} 
Komponente & P 17 & P 117 & P 311 & P 338 \\
\hline
\end{tabular}

\begin{tabular}{lrrrr}
\hline Weichschaumstoff $(\mathrm{g})$ & 430 & 26500 & 1720 & 430 \\
\hline Dipropylenglykol $(\mathrm{g})$ & 140 & 9100 & 1120 & 80 \\
\hline Hexapropylenglykol $(\mathrm{g})$ & & & & 285 \\
\hline Di-n-butylamin $(\mathrm{g})$ & 16 & 1650 & 64 & 10 \\
\hline Hydroxylzahl $(\mathrm{mg} \mathrm{KOH} / \mathrm{g})$ & 224 & 224 & 356 & 173 \\
\hline Viskosität (mPas, $\left.25^{\circ} \mathrm{C}\right)$ & 8120 & 8100 & 3370 & 9560 \\
\hline Aminzahl (mg KOH/g) & 51 & 39 & 48 & 36
\end{tabular}

Wie damit gezeigt wird, konnten Recyclat-Polyole in einem großen Hydroxylzahlbereich bei Viskositäten, die den üblichen Viskositäten der Polyurethanvorprodukte entsprechen, hergestellt werden. Die inzwischen dargestellte Palette an reaktiven Polyolen umfasst einen Hydroxylzahlbereich von 80 bis 400 , wovon die folgende Tabelle wiederum nur eine Auswahl darstellt:

\begin{tabular}{lrrrrrr} 
Parameter & P 318 & P 338 & P 326 & P 223 & P 225 & P222 \\
\hline $\begin{array}{l}\text { Hydroxylzahl } \\
\text { (mg KOH/g) }\end{array}$ & 81 & 173 & 210 & 283 & 328 & 382 \\
\hline $\begin{array}{l}\text { Viskosität } \\
\left.\text { (mPas, } 25^{\circ} \mathrm{C}\right)\end{array}$ & 23.500 & 9560 & 2560 & 7860 & 7700 & 1950 \\
\hline $\begin{array}{l}\text { Aminzahl } \\
\text { (mg KOH/g) }\end{array}$ & 44 & 36 & 17 & 43 & 43 & 31 \\
\hline $\begin{array}{l}\text { Wassergehalt } \\
(\%)\end{array}$ & 0,06 & 0,07 & 0,07 & 0,03 & 0,05 & 0,06 \\
\hline & & & & & &
\end{tabular}

Die Kombination verschiedener Glykole erlaubt es, Recyclat-Polyole mit den für bestimmte Anforderungen benötigten Eigenschaften herzustellen. Die Aminzahl der Polyole resultiert dabei im wesentlichen aus Resten der verwendeten Reaktanden und lässt sich durch anschließendes Entgasen am Rotationsverdampfer $\left(120^{\circ} \mathrm{C} /\right.$ $25 \mathrm{mPa} / 2 \mathrm{~h}$ ) reduzieren. Die Reaktivität der RecyclatPolyole ist gegenuiber Isocyanaten sehr hoch, so dass die Topfzeiten ohne Zusatz von Katalysator im Bereich von 30 bis 90 Sekunden liegen. Eine Verzögerung der Reaktion kann durch die Zugabe von 1 bis 3 Gew.-\% Acetylaceton erreicht werden.

Das wesentliche Anwendungsgebiet der Recyclat-Polyole sollten elastische Beschichtungen sein. Zunächst wurden jedoch die Polyole mit den Hydroxylzahlen uiber 300 auch zur Herstellung von Schaumstoffen verwendet (Standardrezeptur). Auf diese Weise konnten Hartschaumstoffe, z. B. aus dem Polyol P 222, hergestellt werden. Bei einer Zielrohdichte von $40 \mathrm{~g} / \mathrm{dm}^{3}$ ergaben sich folgende Eigenschaften:

Rohdichte $\left(\mathrm{g} / \mathrm{dm}^{3}\right)$ 36,4

Druckfestigkeit $\left(\mathrm{kN} / \mathrm{mm}^{2}\right) \quad 2,87$

Dimensionsstabilität $\left(24 \mathrm{~h} / 140{ }^{\circ} \mathrm{C}\right) \ldots+1,6 \%$

Bei Verwendung der Recyclat-Polyole mit niedrigeren Hydroxylzahlen und niedrigeren Isocyanatindices werden halbharte Schaumstoffe erhalten. Diese Schaumstoffe wurden zunächst im Dichtebereich $60 \mathrm{~g} / \mathrm{dm}^{3}$ hergestellt, um die Eignung der Polyole für diesen Einsatzzweck nachzuweisen.

Aus den Recyclat-Polyolen wurden in erster Linie halbharte Folien als Simulation für Reaktivbeschichtungen auf dem Mathis LabCoater hergestellt. Die Folien wurden nach der Standardformulierung mit Isocyanatindices zwischen 90 und 75 mit dem Messerrakel auf Silikontrennpapier gezogen und bei $90{ }^{\circ} \mathrm{C}$ in 25 Minuten gehärtet. Als Beispiele seien zwei Formulierungen auf der Basis der Recyclatpolyole P 223 und P 224 (Hydroxylzahl $272 \mathrm{mg} \mathrm{KOH} / \mathrm{g}$ ) dargestellt:

\begin{tabular}{lrr} 
Parameter & P 223 & P 224 \\
\hline Polyol $(\mathrm{g})$ & 98,0 & 98,0 \\
\hline Acetylaceton $(\mathrm{g})$ & 1,65 & 1,65 \\
\hline Vinycene 400 & 0,35 & 0,35 \\
\hline Lupranat $® \mathrm{M} \mathrm{20} \mathrm{A}$ & 59,2 & 63,2 \\
\hline Zugfestigkeit kN/mm & 16,9 & 16,5 \\
\hline Bruchdehnung (\%) & 72,8 & 78,7 \\
\hline Shore-A-Härte & 91 & 95 \\
\hline
\end{tabular}

Die Spannungs-Dehnungs-Kurven der beiden hier beschriebenen Folien (Normkleinstäbe mit 40 mm Steglänge und $4 \times 4 \mathrm{~mm}$ Querschnitt) zeigen im Bereich bis ca. $20 \%$ Dehnung ein Hooke'sches Verhalten durch einen nahezu linearen Anstieg der Spannung uiber der Dehnung. Danach folgt ein ausgeprägtes Plateaugebiet bis unmittelbar vor dem Bruch der Probe (Abb. 1). Das Hooke'sche Verhalten im Bereich der niedrigen Dehnungswerte ist auf die Struktur der Polyurethane zurückzuführen, die auf einem langkettigen Polyether und kurzkettigen Diolen basieren. Die relativ breite Plateauphase wird durch die Umlagerung der Polyetherketten im Weichsegment und ihr Gleiten aneinander hervorgerufen, die vernetzte Hartsegmentphase spielt hierbei keine Rolle. Die Reduzierung der Menge an kurzkettigem Diol (P 224) fuihrt zu einer weiteren Verbesserung des elastischen Verhaltens (Abb. 2).

Die Relaxationsspektren der Polyurethane aus den Recycling-Polyolen zeigen einige Besonderheiten gegenüber einem typischen Polyurethan-Elastomeren, die auf die Zusammensetzung der Polyole und das spezielle Herstellungsverfahren zurückzuführen sind. Auf Grund der 


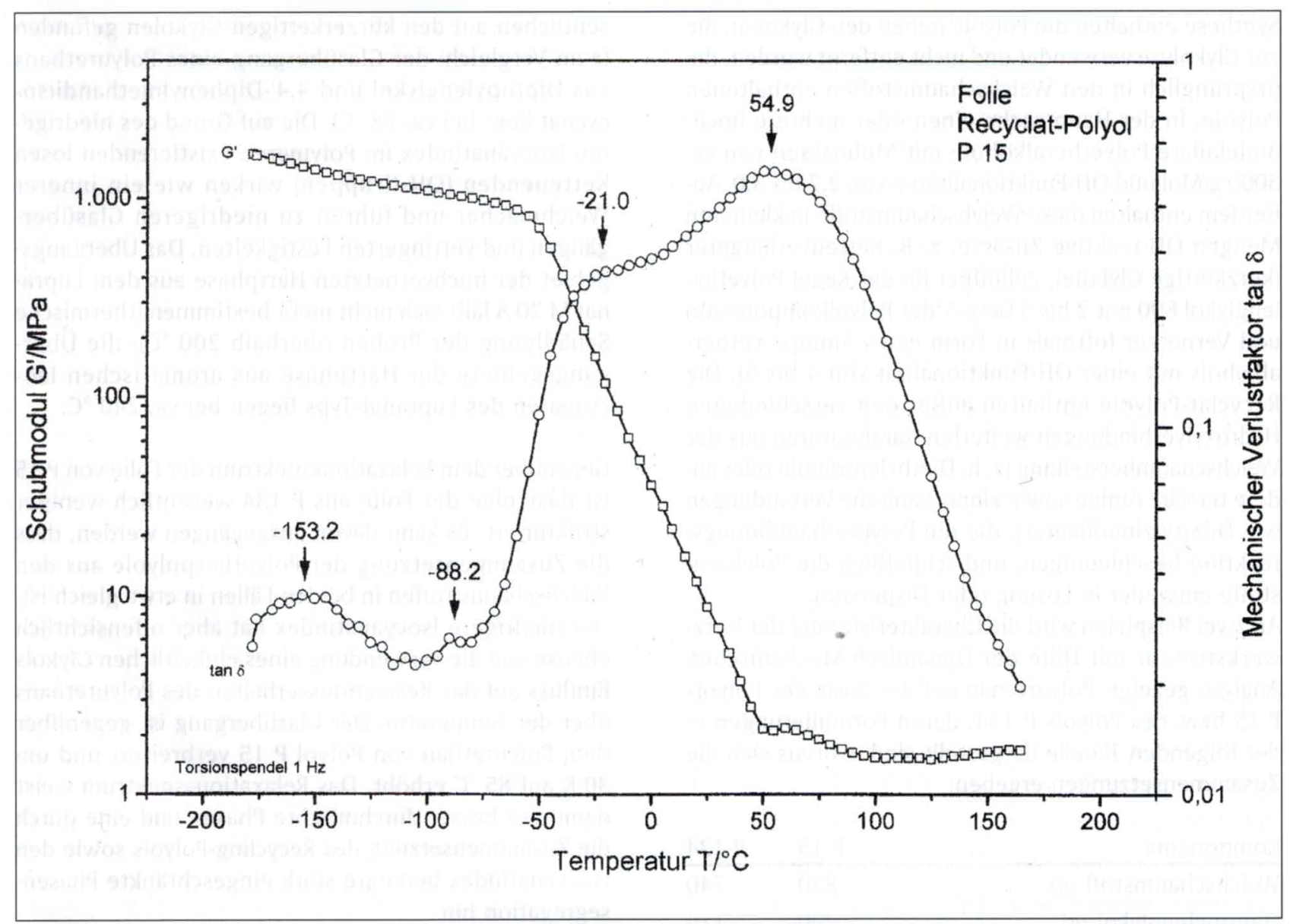

Abb. 1: DMA-Messung einer $4 \mathrm{~mm}$ Folie des Polyurethans aus Polyol P 15 und Lupranat $\mathrm{M} 20 \mathrm{~A}$ zwischen $-170{ }^{\circ} \mathrm{C}$ und $+170^{\circ} \mathrm{C}$ (Myrenne Torsionspendel, Meßfrequenz $1 \mathrm{~Hz}$ )

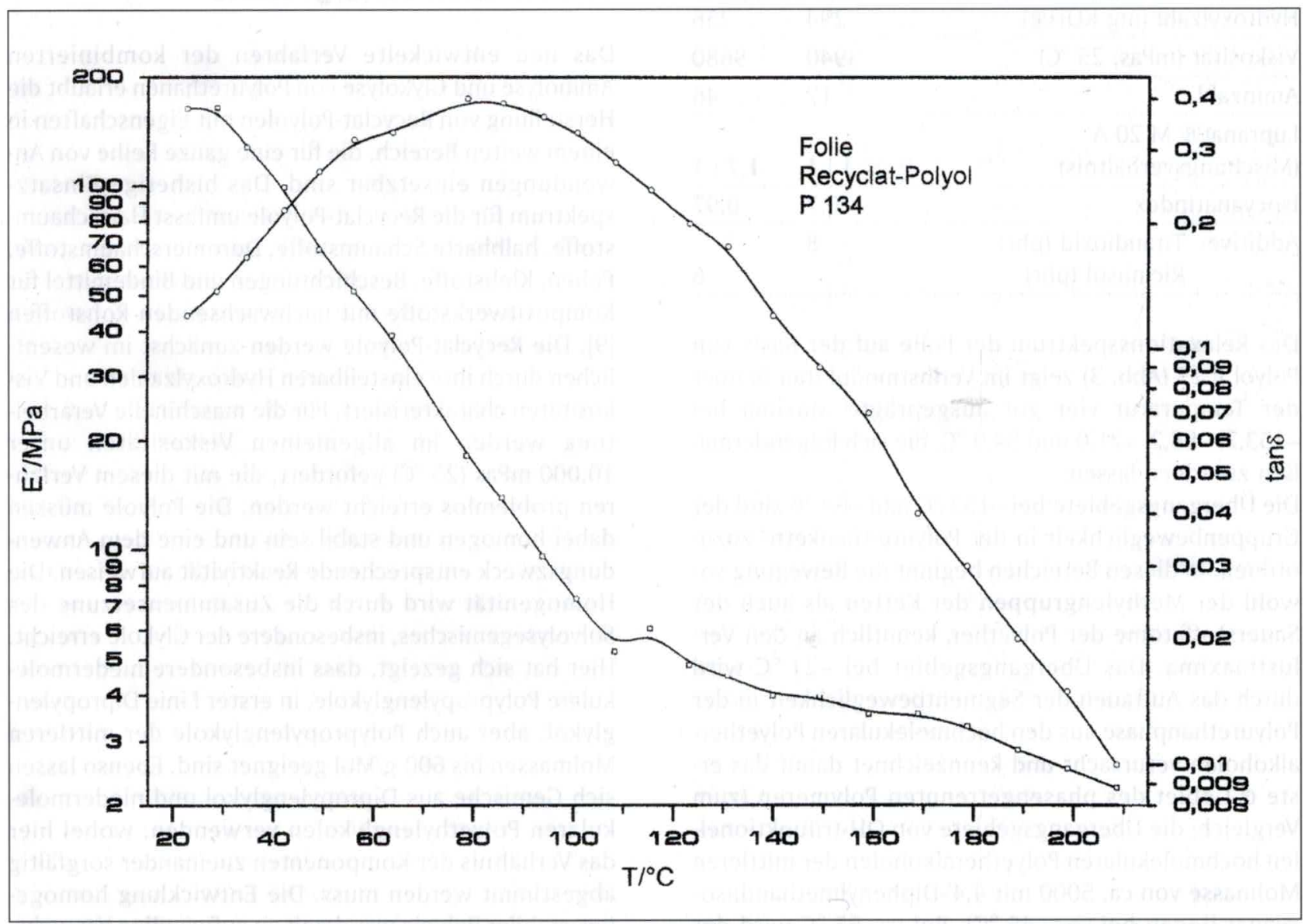

Abb. 2: DMA-Messung einer $4 \mathrm{~mm}$ Folie des Polyurethans aus Polyol P 134 und Lupranat M 20 A zwischen $+20^{\circ} \mathrm{C}$ und $+210^{\circ} \mathrm{C}$ (Rheovibron) 
Synthese enthalten die Polyole neben den Glykolen, die zur Glykolyse verwendet und nicht entfernt wurden, die ursprünglich in den Weichschaumstoffen enthaltenen Polyole, in der Hauptsache einen oder mehrere hochmolekulare Polyetheralkohole mit Molmassen von ca. $5000 \mathrm{~g} / \mathrm{Mol}$ und $\mathrm{OH}-$ Funktionalitäten von 2,7 bis 3,0. Außerdem enthalten diese Weichschaumstoffe in kleineren Mengen $\mathrm{OH}$-reaktive Zusätze, z. B. Kettenverlängerer (kurzkettige Glykole), Zellöffner (in der Regel Polyethylenglykol 600 mit 2 bis 5 Gew.-\% der Polyolkomponente) und Vernetzer (oftmals in Form eines Aminpolyether-

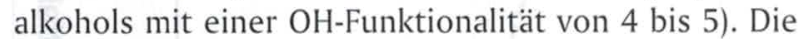
Recyclat-Polyole enthalten außer den verschiedenen Hydroxylverbindungen weiterhin Katalysatoren aus der Weichschaumherstellung (z. B. Diethylentriamin oder andere tertiäre Amine sowie zinnorganische Verbindungen wie Dibutylzinndilaurat), die die Polyurethanbildungsreaktion beschleunigen, und schließlich die Polyharnstoffe entweder in Lösung oder Dispersion.

An zwei Beispielen wird die Charakterisierung der Netzwerkstruktur mit Hilfe der Dynamisch-Mechanischen Analyse gezeigt: Polyurethan auf der Basis des Polyols P 15 bzw. des Polyols P 134, deren Formulierungen in der folgenden Tabelle dargestellt sind, woraus sich die Zusammensetzungen ergeben:

\begin{tabular}{|c|c|c|}
\hline Komponente & P 15 & P 134 \\
\hline Weichschaumstoff (g) & 850 & 740 \\
\hline Dipropylenglykol (g) & 290 & 119 \\
\hline Polyethylenglykol 400 (g) & - & 119 \\
\hline Di-n-butylamin (g) & 53 & 29,5 \\
\hline Hydroxylzahl (mg KOH/g) & 294 & 256 \\
\hline Viskosität (mPas, $25^{\circ} \mathrm{C}$ ) & 3940 & 9680 \\
\hline Aminzahl & 17 & 46 \\
\hline $\begin{array}{l}\text { Lupranat }{ }^{\circledR} \text { M } 20 \text { A } \\
\text { (Mischungsverhältnis) }\end{array}$ & $1: 1$ & $1,7: 1$ \\
\hline Isocyanatindex & 0,7 & 0,97 \\
\hline $\begin{aligned} \text { Additive: } & \text { Titandioxid (phr) } \\
& \text { Ricinusöl (phr) }\end{aligned}$ & 8 & \\
\hline
\end{tabular}

Das Relaxationsspektrum der Folie auf der Basis von Polyol P 15 (Abb. 3) zeigt im Verlustmodul (tan $\delta$ ) iiber der Temperatur vier gut ausgeprägte Maxima bei $-153,2 ;-88,2 ;-21,0$ und $54,9^{\circ} \mathrm{C}$, die sich folgendermaßen zuordnen lassen:

Die Übergangsgebiete bei $-153^{\circ} \mathrm{C}$ und $-88^{\circ} \mathrm{C}$ sind der Gruppenbeweglichkeit in der Polyure-thankette zuzuordnen. In diesen Bereichen beginnt die Bewegung sowohl der Methylengruppen der Ketten als auch der Sauerstoffatome der Polyether, kenntlich an den Verlustmaxima. Das Übergangsgebiet bei $-21{ }^{\circ} \mathrm{C}$ wird durch das Auftauen der Segmentbeweglichkeit in der Polyurethanphase aus den hochmolekularen Polyetheralkoholen verursacht und kennzeichnet damit das erste $\alpha$-Gebiet des phasengetrennten Polymeren (zum Vergleich: die Übergangsgebiete von $\mathrm{OH}$-trifunktionellen hochmolekularen Polyetheralkoholen der mittleren Molmasse von ca. 5000 mit 4,4'-Diphenylmethandiisocyanat liegen bei ca. $-45^{\circ} \mathrm{C}$ ). Bei ca. $55^{\circ} \mathrm{C}$ wird der Glasübergang der Polyurethanphase basierend im we- sentlichen auf den kürzerkettigen Glykolen gefunden (zum Vergleich: der Glasübergang eines Polyurethans aus Dipropylenglykol und 4,4'-Diphenylmethandiisocyanat liegt bei ca. $78^{\circ} \mathrm{C}$ ). Die auf Grund des niedrigeren Isocyanatindex im Polymeren existierenden losen Kettenenden (OH-Gruppen) wirken wie ein innerer Weichmacher und führen zu niedrigeren Glasübergängen und verringerten Festigkeiten. Das Übergangsgebiet der hochvernetzten Hartphase aus dem Lupranat M 20 A läßt sich nicht mehr bestimmen (thermische Schädigung der Proben oberhalb $200{ }^{\circ} \mathrm{C}$ ); die Übergangsgebiete der Hartphase aus aromatischen Isocyanaten des Lupranat-Typs liegen bei ca. $240{ }^{\circ} \mathrm{C}$.

Gegenüber dem Relaxationsspektrum der Folie von P 15 ist dasjenige der Folie aus P 134 wesentlich weniger strukturiert. Es kann davon ausgegangen werden, dass die Zusammensetzung der Polyetherpolyole aus den Weichschaumstoffen in beiden Fällen in etwa gleich ist. Der niedrigere Isocyanatindex hat aber offensichtlich ebenso wie die Verwendung eines einheitlichen Glykols Einfluss auf das Relaxationsverhalten des Polyurethans über der Temperatur. Der Glasübergang ist gegenüber dem Polyurethan von Polyol P 15 verbreitert und um $30 \mathrm{~K}$ auf $85^{\circ} \mathrm{C}$ erhöht. Das Relaxationsspektrum weist damit auf besser durchmischte Phasen und eine durch die Zusammensetzung des Recycling-Polyols sowie den Isocyanatindex bedingte stark eingeschränkte Phasensegregation hin.

\section{Diskussion der Ergebnisse}

Das neu entwickelte Verfahren der kombinierten Aminolyse und Glykolyse von Polyurethanen erlaubt die Herstellung von Recyclat-Polyolen mit Eigenschaften in einem weiten Bereich, die für eine ganze Reihe von Anwendungen einsetzbar sind. Das bisherige Einsatzspektrum für die Recyclat-Polyole umfasst Hartschaumstoffe, halbharte Schaumstoffe, Duromerschaumstoffe, Folien, Klebstoffe, Beschichtungen und Bindemittel für Kompositwerkstoffe mit nachwachsenden Rohstoffen [9]. Die Recyclat-Polyole werden zunächst im wesentlichen durch ihre einstellbaren Hydroxylzahlen und Viskositäten charakterisiert. Für die maschinelle Verarbeitung werden im allgemeinen Viskositäten unter $10.000 \mathrm{mPas}\left(25^{\circ} \mathrm{C}\right)$ gefordert, die mit diesem Verfahren problemlos erreicht werden. Die Polyole müssen dabei homogen und stabil sein und eine dem Anwendungszweck entsprechende Reaktivität aufweisen. Die Homogenität wird durch die Zusammensetzung des Solvolysegemisches, insbesondere der Glykole erreicht. Hier hat sich gezeigt, dass insbesondere niedermolekulare Polypropylenglykole, in erster Linie Dipropylenglykol, aber auch Polypropylenglykole der mittleren Molmassen bis $600 \mathrm{~g} / \mathrm{Mol}$ geeignet sind. Ebenso lassen sich Gemische aus Dipropylenglykol und niedermolekularen Polyethylenglykolen verwenden, wobei hier das Verhältnis der Komponenten zueinander sorgfältig abgestimmt werden muss. Die Entwicklung homogener, stabiler Polyole war damit an aufwendige Versuchsserien geknuipft. 


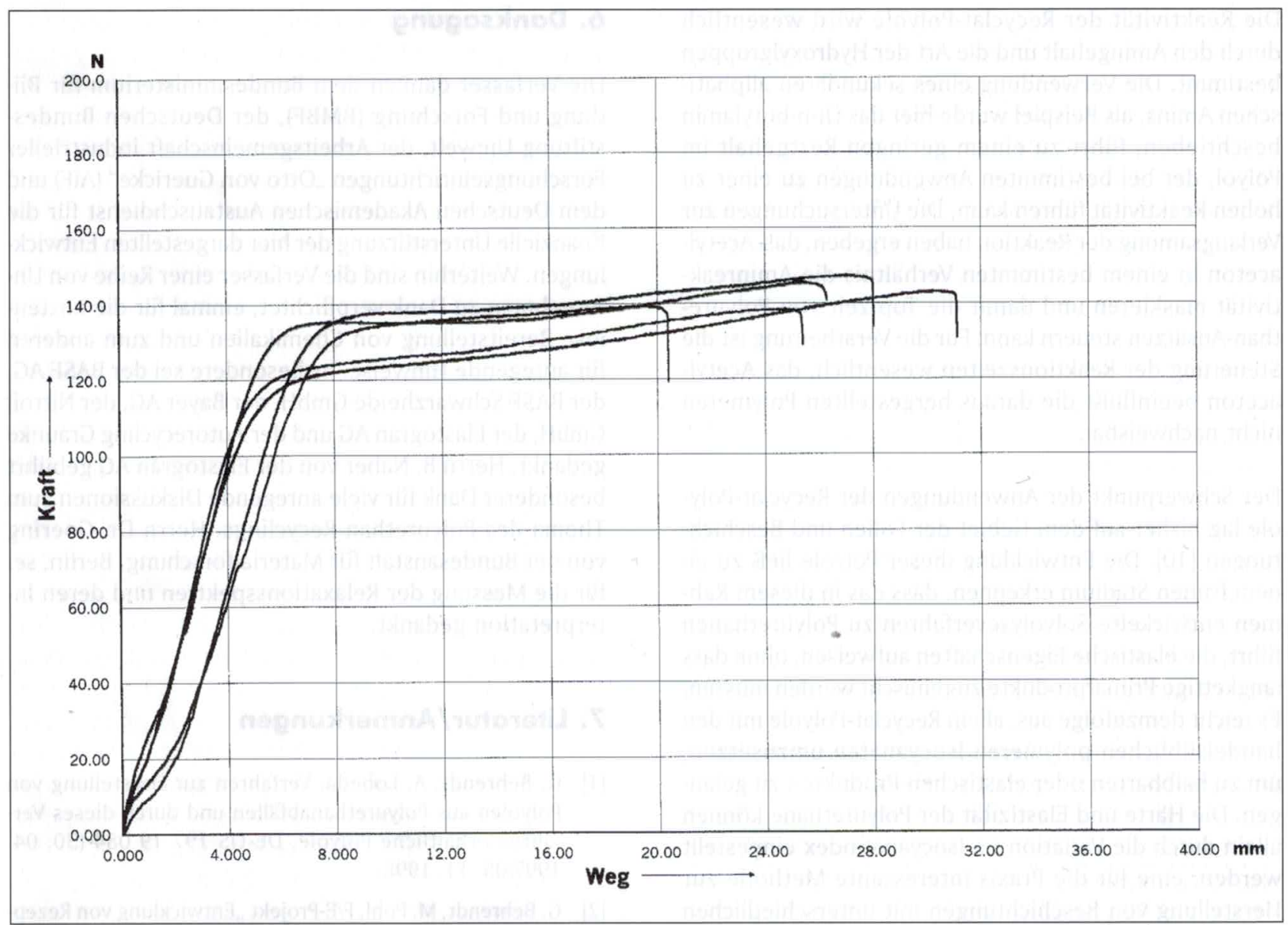

Abb. 3: Spannungs-Dehnungs-Messungen an 4 mm-Proben aus Polyol P 223 Lupranat M 20 A (Steglänge $40 \mathrm{~mm}$, Breite des Steges $4 \mathrm{~mm}$ ), Messung bei $25^{\circ} \mathrm{C}$

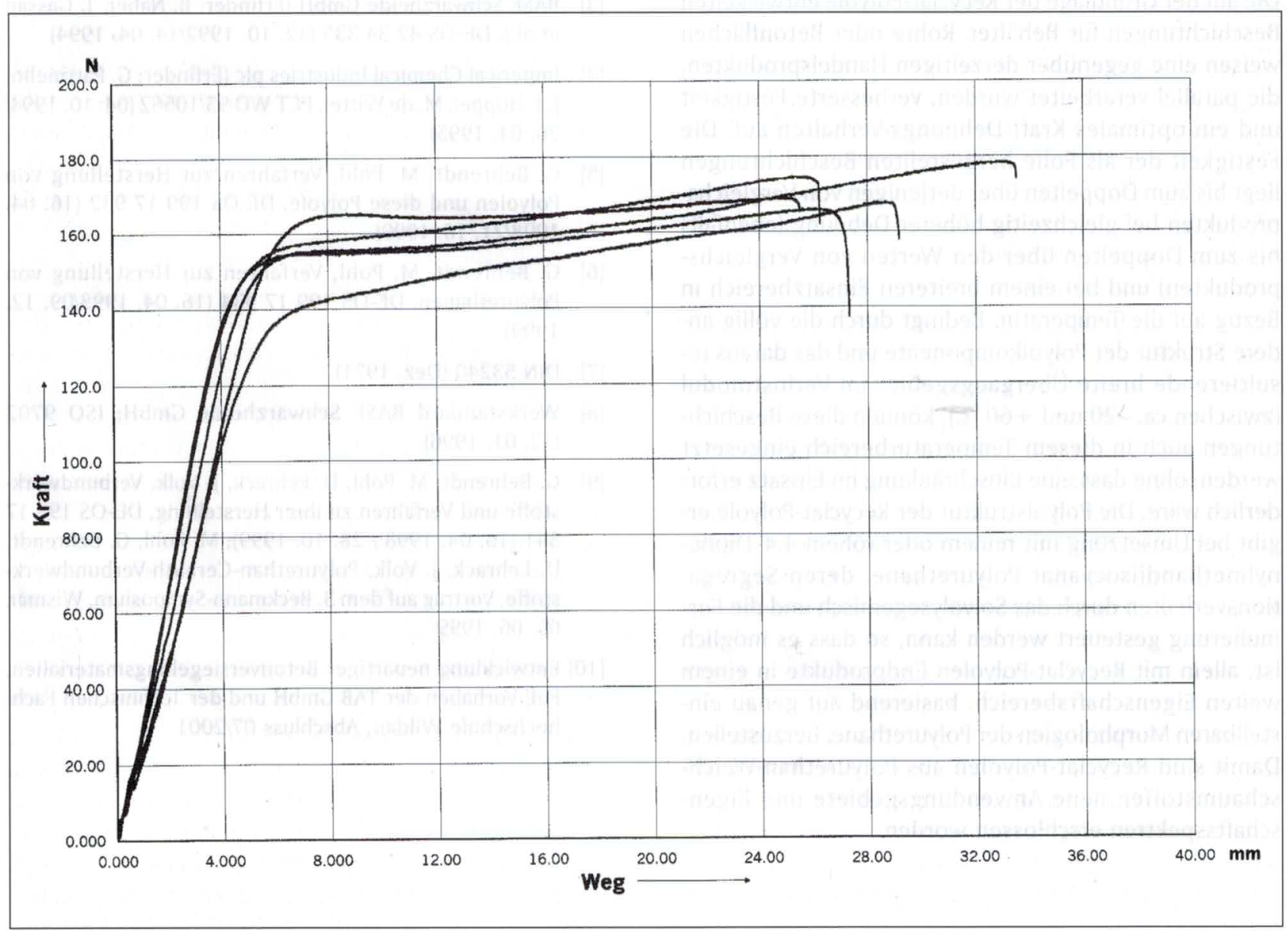

Abb. 4: Spannungs-Dehnungs-Messungen an 4 mm-Proben aus Polyol P 224 Lupranat M 20 A (Steglänge $40 \mathrm{~mm}$, Breite des Steges $4 \mathrm{~mm}$ ), Messung bei $25^{\circ} \mathrm{C}$ 
Die Reaktivität der Recyclat-Polyole wird wesentlich durch den Amingehalt und die Art der Hydroxylgruppen bestimmt. Die Verwendung eines sekundären aliphatischen Amins, als Beispiel wurde hier das Di-n-butylamin beschrieben, führt zu einem geringen Restgehalt im Polyol, der bei bestimmten Anwendungen zu einer zu hohen Reaktivität fuihren kann. Die Untersuchungen zur Verlangsamung der Reaktion haben ergeben, daß Acetylaceton in einem bestimmten Verhältnis die Aminreaktivität maskieren und damit die Topfzeit von Polyurethan-Ansätzen steuern kann. Für die Verarbeitung ist die Steuerung der Reaktionszeiten wesentlich, das Acetylaceton beeinflußt die daraus hergestellten Polymeren nicht nachweisbar.

Der Schwerpunkt der Anwendungen der Recyclat-Polyole lag bisher auf dem Gebiet der Folien und Beschichtungen [10]. Die Entwicklung dieser Polyole ließ zu einem frühen Stadium erkennen, dass das in diesem Rahmen entwickelte Solvolyseverfahren zu Polyurethanen führt, die elastische Eigenschaften aufweisen, ohne dass langkettige Primärprodukte zugemischt werden müssen. Es reicht demzufolge aus, allein Recyclat-Polyole mit den handelsüblichen polymeren Isocyanaten umzusetzen, um zu halbharten oder elastischen Produkten zu gelangen. Die Härte und Elastizität der Polyurethane können allein durch die Variation im Isocyanatindex eingestellt werden, eine für die Praxis interessante Methode zur Herstellung von Beschichtungen mit unterschiedlichen Eigenschaften bei gleichem Polyurethan-System.

Die auf der Grundlage der Recyclat-Polyole entwickelten Beschichtungen für Behälter, Rohre oder Betonflächen weisen eine gegenüber derzeitigen Handelsprodukten, die parallel verarbeitet wurden, verbesserte Festigkeit und ein optimales Kraft-Dehnungs-Verhalten auf. Die Festigkeit der als Folie hergestellten Beschichtungen liegt bis zum Doppelten über derjenigen von Vergleichsprodukten bei gleichzeitig höherer Dehnung (ebenfalls bis zum Doppelten über den Werten von Vergleichsprodukten) und bei einem breiteren Einsatzbereich in Bezug auf die Temperatur. Bedingt durch die völlig andere Struktur der Polyolkomponente und das daraus resultierende breite Übergangsgebiet im Verlustmodul (zwischen ca. -20 und $+60{ }^{\circ} \mathrm{C}$ ), können diese Beschichtungen auch in diesem Temperaturbereich eingesetzt werden, ohne dass eine Einschränkung im Einsatz erforderlich wäre. Die Polyolstruktur der Recyclat-Polyole ergibt bei Umsetzung mit reinem oder rohem 4,4'-Diphenylmethandiisocyanat Polyurethane, deren Segregationsverhalten durch das Solvolysegemisch und die Formulierung gesteuert werden kann, so dass es möglich ist, allein mit Recyclat-Polyolen Endprodukte in einem weiten Eigenschaftsbereich, basierend auf genau einstellbaren Morphologien der Polyurethane, herzustellen. Damit sind Recyclat-Polyolen aus Polyurethan-Weichschaumstoffen neue Anwendungsgebiete und Eigenschaftsspektren erschlossen worden.

\section{Danksagung}

Die Verfasser danken dem Bundesministerium für Bildung und Forschung (BMBF), der Deutschen Bundesstiftung Umwelt, der Arbeitsgemeinschaft industrieller Forschungseinrichtungen „Otto von Guericke“ (AiF) und dem Deutschen Akademischen Austauschdienst für die finanzielle Unterstuitzung der hier dargestellten Entwicklungen. Weiterhin sind die Verfasser einer Reihe von Unternehmen zu Dank verpflichtet, einmal für die kostenlose Bereitstellung von Chemikalien und zum anderen für anregende Hinweise. Insbesondere sei der BASF AG, der BASF Schwarzheide GmbH, der Bayer AG, der Nitroil $\mathrm{GmbH}$, der Elastogran AG und der Autorecycling Graunke gedankt. Herrn B. Naber von der Elastogran AG gebührt besonderer Dank für viele anregende Diskussionen zum Thema des Polyurethan-Recyclings. Herrn Dr. Goering von der Bundesanstalt für Materialforschung, Berlin, sei für die Messung der Relaxationsspektren und deren Interpretation gedankt.

\section{Literatur/Anmerkungen}

[1] G. Behrendt, A. Lobeda, Verfahren zur Herstellung von Polyolen aus Polyurethanabfallen und durch dieses Verfahren erhältliche Polyole, DE-OS 19719084 (30. 04. 1997/05. 11. 1998)

[2] G. Behrendt, M. Pohl, F/E-Projekt „Entwicklung von Rezepturen für Polyurethane auf Basis von Recyclaten“, aFuEVorhaben des BMBF, Abschlußbericht 1998

[3] BASF Schwarzheide GmbH (Erfinder: B. Naber, T. Gassan et al.), DE-OS 4234335 (12. 10. 1992/14. 04. 1994)

[4] Imperical Chemical Industries plc (Erfinder: G. Parrinello, J. F. Hopper, M. de Witte), PCT WO 95/10562 (04. 10. 1994/ 20. 04. 1995)

[5] G. Behrendt, M. Pohl, Verfahren zur Herstellung von Polyolen und diese Polyole, DE-OS 19917932 (16. 04. 1998/21. 10. 1999)

[6] G. Behrendt, M. Pohl, Verfahren zur Herstellung von Polyurethanen, DE-OS 19917934 (16. 04. 1998/09. 12. 1999)

[7] DIN 53240 (Dez. 1971)

[8] Werkstandard BASF Schwarzheide GmbH; ISO 9702 (12.01. 1996)

[9] G. Behrendt, M. Pohl, U. Lehrack, J. Volk, Verbundwerkstoffe und Verfahren zu ihrer Herstellung, DE-OS 19817 541 (16. 04. 1998 / 28. 10. 1999); M. Pohl, G. Behrendt, U. Lehrack, J. Volk, Polyurethan-Ceralith-Verbundwerkstoffe, Vortrag auf dem 3. Beckmann-Symposium, Wismar, 05. 06. 1999

[10] Entwicklung neuartiger Betonversiegelungsmaterialien, FuE-Vorhaben der TAB GmbH und der Technischen Fachhochschule Wildau, Abschluss 07/2001 


\section{Autoren}

Prof. Dr. rer. nat. Gerhard Behrendt

Technische Fachhochschule Wildau

Institut für Abfallwirtschaft/Bodensanierung

Tel. (0 33 75) 508-591

E-Mail: behrendt@vt.tfh-wildau.de

Dipl.-Chem. Hannelore Huth

Technische Fachhochschule Wildau

Institut für Kreislaufwirtschaft

Tel. (0 33 75) 508-225

cand. Ing. Svetlana Ivanyi

Universität für Chemische Technologie und

Metallurgie Sofia

Institut für Schuh- und Lederindustrie

Bul. Sv. Kl. Ohridski 8

1756 Sofia, Bulgarien

\section{Dipl.-Ing. Hagen Koch}

TAB Tankanlagen- und Behälterbau GmbH

Feldstraße 2

03253 Doberlug-Kirchhain

Tel. (03 53 22) 38200

\section{Dipl.-Chem. Martin Pohl}

Technische Fachhochschule Wildau

Institut für Kreislaufwirtschaft

Tel. (0 33 75) 508-156

Dipl.-Ing. Karl-Heinz Schmidt

Technische Fachhochschule Wildau

Institut fuir Kreislaufwirtschaft

Tel. (0 33 75) 508-176

Dipl.-Ing. Oskar Stürmer

EPIK Entwicklung und Produktion Innovativer Kunststoff-

teile $\mathrm{GmbH}$

Lindenstraße 61

15517 Fürstenwalde

Tel. (0 33 61) 6930109 